SLAC-PUB-9088

December 2001

\title{
Operational Experience with the DIRC Detector*
}

I. Adam, ${ }^{1}$ R. Aleksan, ${ }^{2}$ D. Aston, ${ }^{1}$ D. Bernard,${ }^{5}$ G. Bonneaud, ${ }^{5}$ P. Bourgeois,${ }^{2}$ F. Brochard, ${ }^{5}$ D.N. Brown, ${ }^{6}$ J. Chauveau, ${ }^{3}$ J. Cohen-Tanugi, ${ }^{5}$ M. Convery, ${ }^{1}$ S. Emery, ${ }^{2}$ S. Ferrag, ${ }^{5}$ A. Gaidot,${ }^{2}$ T. Haas,${ }^{1}$ T. Hadig, ${ }^{1}$ G. Hamel de Monchenault, ${ }^{2}$ C. Hast,${ }^{4}$ A. Hoecker, ${ }^{4}$ R.W. Kadel,${ }^{6}$ J. Kadyk ${ }^{6}$ M. Krishnamurthy,${ }^{8}$ H. Lacker, ${ }^{4}$ G.W. London ${ }^{2}$ A. Lu, ${ }^{7}$ A.-M. Lutz, ${ }^{4}$ G. Lynch, ${ }^{6}$ G. Mancinelli, ${ }^{9}$ N. Mayer,${ }^{2}$ B.T. Meadows, ${ }^{9}$ Ll.M. Mir, ${ }^{6}$ D. Muller, ${ }^{1}$ J. Ocariz ${ }^{3}$ I. Ofte ${ }^{9}{ }^{\text {S. Plaszczynski, }}{ }^{4}$ M. Pripstein, ${ }^{6}$ B.N. Ratcliff,,${ }^{1}$ L. Roos,${ }^{3}$ M.-H. Schune, ${ }^{4}$ J. Schwiening, ${ }^{1 \dagger}$ V. Shelkov, ${ }^{6}$ M.D. Sokoloff, ${ }^{9}$ S. Spanier, ${ }^{1}$ J. Stark, ${ }^{3}$ A.V. Telnov, ${ }^{6}$ Ch. Thiebaux,${ }^{5}$ G. Vasileiadis,${ }^{5}$ G. Vasseur ${ }^{2}$ J. Va'vra, ${ }^{1}$ M. Verderi, ${ }^{5}$ W.A. Wenzel, ${ }^{6}$ R.J. Wilson, ${ }^{8}$ G. Wormser, ${ }^{4}$ Ch. Yéche, ${ }^{2}$ S. Yellin, ${ }^{7}$ M. Zito. ${ }^{2}$

${ }^{1}$ Stanford Linear Accelerator Center, Stanford, CA 94309, USA.

${ }^{2}$ CEA, DAPNIA, CE-Saclay, F-91191, Gif-sur-Yvette Cedex, France.

${ }^{3}$ LPNHE des Universités Paris 6 et Paris 7, Tour 33, Bc 200, 4 Place Jussieu, F-75252, Paris, Cedex 05, France.

${ }^{4}$ LAL Orsay, Universite Paris Sud, Batiment 200, F-91405 Orsay Cedex, France.

${ }^{5}$ LPNHE de l'Ecole Polytechnique, Route de Saclay, F-91128 Palaiseau Cedex, France.

${ }^{6}$ Lawrence Berkeley National Laboratory, One Cyclotron Road, Berkeley, CA 94720, USA.

${ }^{7}$ Dept. of Physics, University of California, Santa Barbara, CA 93106, USA.

${ }^{8}$ Dept. of Physics, Colorado State University, Fort Collins, CO 80523, USA.

${ }^{9}$ Dept. of Physics, University of Cincinnati, Cincinnati, OH 45221, USA.

\begin{abstract}
The DIRC, a novel type of Cherenkov ring imaging device, is the primary hadronic particle identification system for the BABAR detector at the asymmetric B-factory, PEP-II at SLAC. It is based on total internal reflection and uses long, rectangular bars made from synthetic fused silica as Cherenkov radiators and light guides. $B_{A} B A R$ began taking data with colliding beams in late spring 1999 . This paper describes the performance of the DIRC during the first 2.5 years of operation.

\author{
Invited talk presented at 2001 IEEE Nuclear Science Symposium \\ San Diego, California, USA \\ Nov 4-Nov 10, 2001 \\ Submitted to IEEE Transactions on Nuclear Science
}

*Work supported by Department of Energy contract DE-AC03-76SF00515.

${ }^{\dagger}$ Speaker and contact: jochen@slac.stanford.edu 


\section{Introduction}

The study of $C P$-violation using hadronic final states of the $B \bar{B}$ meson system requires the ability to tag the flavor of one of the $B$ mesons via the cascade decay $b \rightarrow c \rightarrow s$, while fully reconstructing the final state of the other over a large region of solid angle and momentum. The momenta of the kaons used for flavor tagging extend up to about $2 \mathrm{GeV} / c$, with most of them below $1 \mathrm{GeV} / c$. On the other hand, pions from the rare two-body decays $B^{0} \rightarrow$ $\pi^{+} \pi^{-}\left(K^{+} \pi^{-}\right)$must be well-separated from kaons. They have momenta between 1.7 and 4.2 $\mathrm{GeV} / c$ with a strong momentum-polar angle correlation between the tracks (higher momenta occur at the more forward angles because of the c.m. system boost) [1]. The Particle Identification (PID) system in BABAR is located inside the calorimeter volume. Therefore, it should be thin and uniform in terms of radiation lengths (to minimize degradation of the calorimeter energy resolution) and small in the radial dimension to reduce the volume, hence, the cost of the calorimeter. Finally, for operation at high luminosity, the PID system needs fast signal response, and should be able to tolerate high backgrounds.

The PID system being used in $B A B A R$ is a new kind of ring-imaging Cherenkov detector called the DIRC [2] (the acronym DiRC stands for Detector of Internally Reflected Cherenkov light). It is designed to be able to provide excellent $\pi / K$ separation for all tracks from $B$ -

meson decays from the pion Cherenkov threshold up to $4.2 \mathrm{GeV} / \mathrm{c}$. PID below $700 \mathrm{MeV} / \mathrm{c}$ exploits primarily the $d E / d x$ measurements in the silicon vertex tracker and drift chamber.

\section{DIRC Concept}

The DIRC is based on the principle that the magnitudes of angles are maintained upon reflection from a flat surface. Figure 1 shows a schematic of the DiRC geometry that illustrates the principles of light production, transport, and imaging. The radiator material of the DiRC is synthetic, fused silica in the form of long, thin bars with rectangular cross section. These bars serve both as radiators and as light pipes for the portion of the light trapped in the radiator by total internal reflection. Fused, synthetic silica (Spectrosil [3]) is chosen because of its resistance to ionizing radiation, its long attenuation length, large index of refraction, small chromatic dispersion within the wavelength acceptance of the DIRC, and because it allows an excellent optical finish on the surfaces of the bars [4].

In the following, the variable $\theta_{c}$ is used to designate the Cherenkov angle, $\phi_{c}$ denotes the azimuthal angle of a Cherenkov photon around the track direction, and $n$ represents the mean index of refraction of fused silica $(n=1.473)$. The Cherenkov angle is given by the familiar relation $\cos \theta_{c}=1 / n \beta(\beta=v / c, v=$ velocity of the particle, $c=$ velocity of light $)$.

For particles with $\beta \approx 1$, some photons will always lie within the total internal reflection limit, and will be transported to either one or both ends of the bar, depending on the particle incident angle. To avoid instrumenting both ends of the bar with photon detectors, a mirror is placed at the forward end, perpendicular to the bar axis, to reflect incident photons to the backward, instrumented end (see Figure 2).

Once photons arrive at the instrumented end, most of them emerge into a water-filled expansion region, called the standoff box. A fused silica wedge at the exit of the bar reflects 


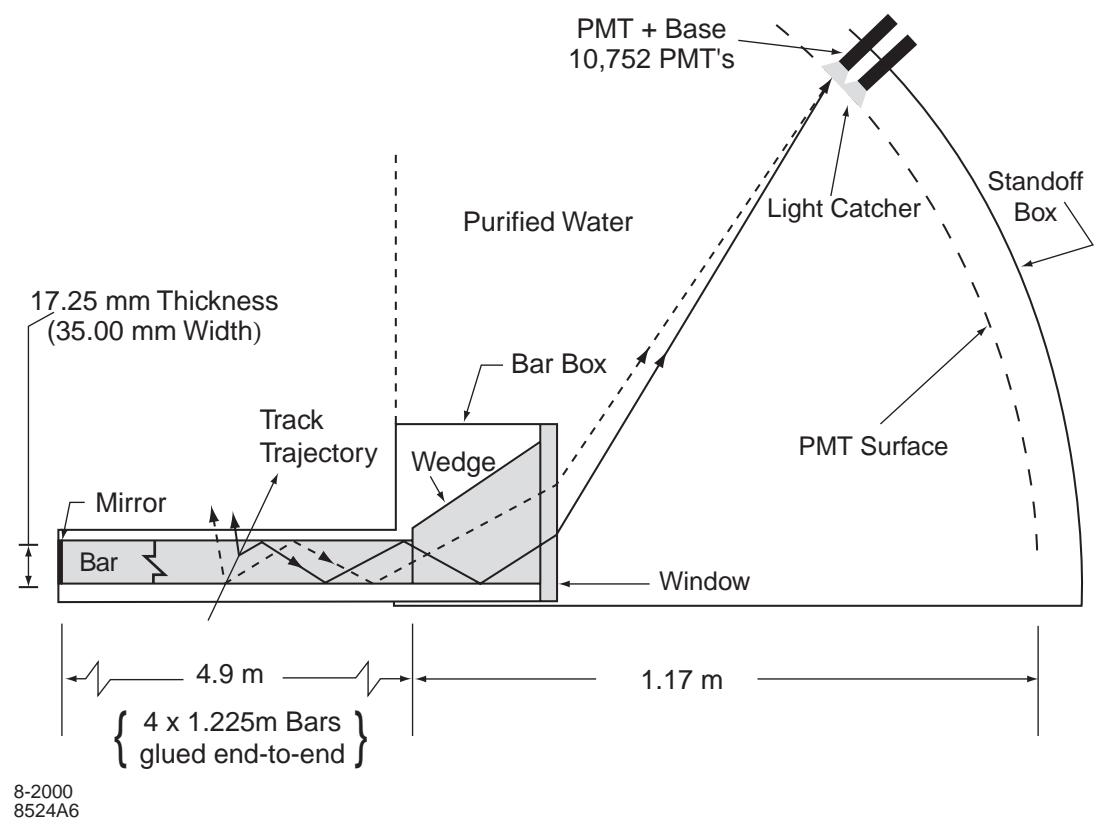

Figure 1: Schematic of the DIRC fused silica radiator bar and imaging region.

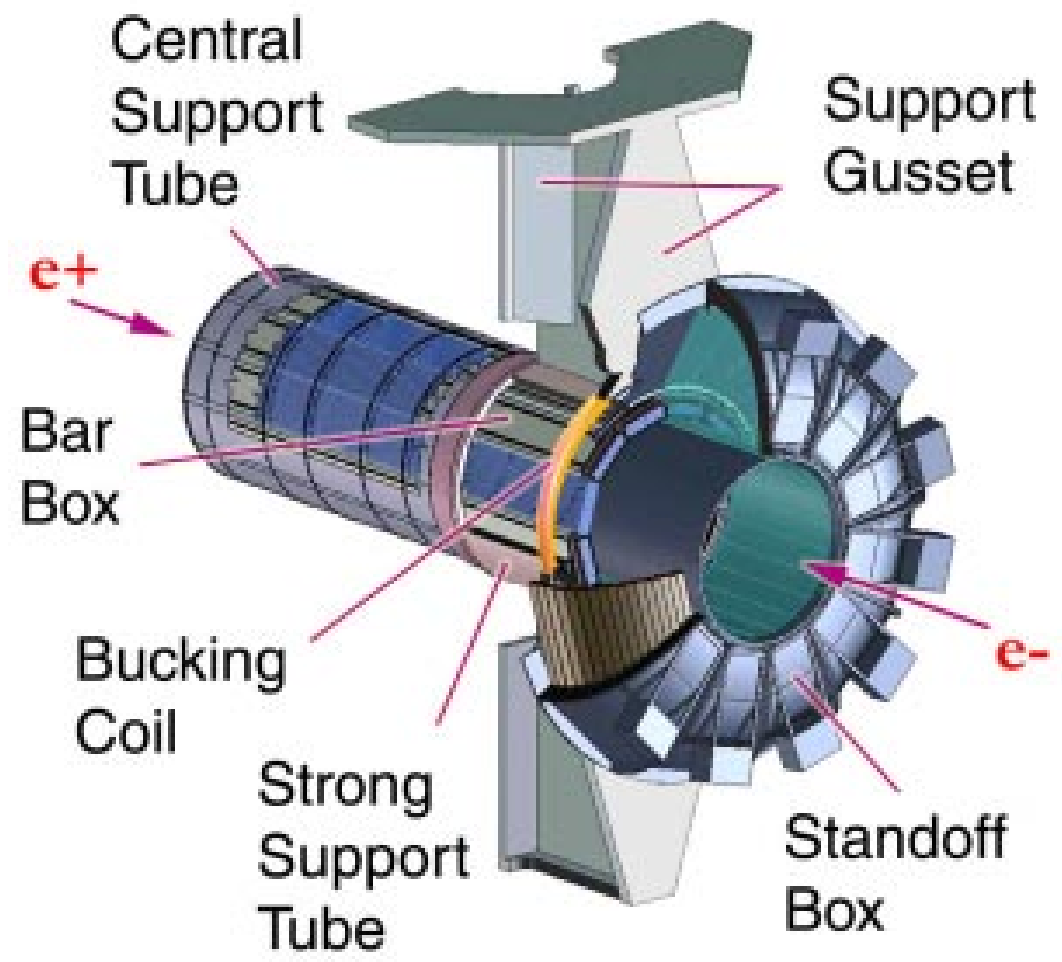

Figure 2: Exploded view of the DIRC mechanical support structure. The iron magnetic shield is not shown. 
photons at large angles relative to the bar axis. It thereby reduces the size of the required detection surface and recovers those photons that would otherwise be lost due to internal reflection at the fused silica/water interface. The photons are detected by an array of densely packed photomultiplier tubes (PMTs), each surrounded by reflecting light catcher cones [5] to capture light which would otherwise miss the active area of the PMT. The PMTs are placed at a distance of about $1.2 \mathrm{~m}$ from the end of the bars. The expected Cherenkov light pattern at this surface is essentially a conic section, where the cone opening-angle is the Cherenkov production angle modified by refraction at the exit from the fused silica window.

The DIRC is intrinsically a three-dimensional imaging device. Photons are focused onto the phototube detection surface via a "pinhole" defined by the exit aperture of the bar, so that the photon propagation angles $\alpha_{x}$ and $\alpha_{y}$ can be measured in two-dimensional space, where $\mathrm{x}$ (bar width) and $\mathrm{y}$ (bar thickness) are the directions transverse to the bar axis. The time taken for the photon to travel down the bar is also related to the photon propagation angle $\left(\alpha_{z}\right)$ with respect to the bar axis. Imaging in the DIRC occurs in all three of these dimensions, by recording the time of the PMT signal. As the track position and angles are known from the tracking system, these three $\alpha$ angles can be used to determine the two angles $\left(\theta_{c}, \phi_{c}\right)$. This over-constraint on the angles is particularly useful in dealing with ambiguities (see below) and high background rates.

The DiRc bars are arranged in a 12-sided polygonal barrel. Because of the beam energy asymmetry (at the $\Upsilon(4 S)$, PEP-II collides $9 \mathrm{GeV}$ electrons on $3.1 \mathrm{GeV}$ positrons), particles are produced preferentially forward in the laboratory. To minimize interference with other detector systems in the forward region, the DIRC photon detector is placed at the backward end. The bars are placed into 12 hermetically sealed containers, called bar boxes, made of very thin aluminum-hexcel panels. Dry nitrogen gas flows through each box, and is monitored for humidity to ensure that the bar box to water interface remains sealed. Each bar box contains 12 bars, for a total of 144 bars. Within a bar box the 12 bars are optically isolated by a $\sim 150 \mu \mathrm{m}$ air gap between neighboring bars, enforced by custom shims made from aluminum foil.

The bars are $17 \mathrm{~mm}$-thick, $35 \mathrm{~mm}$-wide, and $4.9 \mathrm{~m}$-long. Each bar is assembled from four $1.225 \mathrm{~m}$ pieces that are glued end-to-end; this length is the longest high-quality bar currently obtainable $[4,6]$. The bars are supported at $600 \mathrm{~mm}$ intervals by small nylon buttons for optical isolation from the bar box. Each bar has a fused silica wedge glued to it at the readout end. The wedge, made of the same material as the bar, is $91 \mathrm{~mm}$-long with very nearly the same width $(33 \mathrm{~mm})$ as the bars and a trapezoidal profile $(27 \mathrm{~mm}$-high at bar end, and $79 \mathrm{~mm}$-high at the light exit end). The bottom of the wedge (see Figure 1) has a slight $(\sim 6 \mathrm{mr})$ upward slope to minimize the displacement of the downward reflected image due to the finite bar thickness. The twelve wedges in a bar box are glued to a common $10 \mathrm{~mm}$-thick fused silica window, that provides the interface and seal to the purified water in the standoff box.

The standoff box is made of stainless steel, consisting of a cone, cylinder, and 12 sectors of PMTs. It contains about 6,000 liters of purified water. Water is used to fill this region because it is inexpensive and has an average index of refraction $(n \approx 1.346)$ reasonably close to that of fused silica, thus minimizing the total internal reflection at silica-water interface. 
Furthermore, its chromaticity index is a close match to that of fused silica, effectively eliminating dispersion at the silica-water interface. The iron gusset supports the standoff box. An iron shield, supplemented by a bucking coil, surrounds the standoff box to reduce the field in the PMT region to below 1 Gauss [7].

The PMTs at the rear of the standoff box lie on a surface that is approximately toroidal. Each of the 12 PMT sectors contains 896 PMTs (ETL model 9125 [8, 9]) with 29 mmdiameter, in a closely packed array inside the water volume. A double o-ring water seal is made between the PMTs and the vessel wall. The PMTs are installed from the inside of the standoff box and connected via a feed-through to a base mounted outside. The hexagonal light catcher cone is mounted in front of the photocathode of each PMT, which results in an effective active surface area light collection fraction of about $90 \%$.

The DIRC occupies $80 \mathrm{~mm}$ of radial space in the central detector volume including supports and construction tolerances, with a total of about 19\% radiation length thickness at normal incidence. The radiator bars subtend a solid angle corresponding to about $94 \%$ of the azimuth and $83 \%$ of the c.m. polar angle cosine.

The distance from the end of the bar to the PMTs is $\sim 1.17 \mathrm{~m}$, which together with the size of the bars and PMTs, gives a geometric contribution to the single photon Cherenkov angle resolution of $\sim 7 \mathrm{mr}$. This is slightly larger than the resolution contribution expected from the photon production (dominated by a $\sim 5.4 \mathrm{mr}$ chromatic term) and transmission dispersions. The overall single photon resolution is estimated to be about $9.5 \mathrm{mr}$.

\section{Operational Issues}

The DIRC was successfully commissioned and attained performance close to that expected from Monte Carlo simulation. The DiRC has been robust and stable, and, indeed, serves also as a background detector for PEP-II tuning. Figure 3 shows a typical di-muon event $\left(e^{+} e^{-} \rightarrow\right.$ $\left.\mu^{+} \mu^{-}\right)$. In addition to the signals caused by the Cherenkov light from the two tracks, about 500 background signals can be seen in the readout window of \pm 300 ns. This background is dominated by low energy photons from the PEP-II machine hitting the standoff box.

The TDC chip [10] used in the DIRC data readout is designed such that a dead time of about $5 \%$ occurs at an input rate of $250 \mathrm{kHz}$. Some care in machine tuning is required to stay under a limit of $250 \mathrm{kHz} /$ tube. To monitor that rate, one PMT in each sector is read out via a scaler. Figure 4 shows the maximum scaler rate as a function of the PEP-II luminosity during data taking in 2000 and 2001. In March 2000 the accelerator operated at a peak luminosity of $1 \times 10^{33} / \mathrm{cm}^{2}$ sec. Figure $4(\mathrm{a})$ shows that, at a value that corresponds to only one third of the design luminosity, the PMT rates reached a level that caused noticeable dead times. Due to those findings, lead shielding was installed in the summer of 2000 around the beam line components near the backward endcap. Initially, localized shielding was added in the form of lead bricks that were stacked around the beam pipe and in front of a large quadrupole. This shielding significantly improved the background situation so that noticeable TDC dead times were reached only at $2.5 \times 10^{33} / \mathrm{cm}^{2} \mathrm{sec}$ (Figure $4(\mathrm{~b})$ ). During the shutdown in January 2001, the localized lead brick shielding was replaced by an engineered, homogeneous lead shielding of $5 \mathrm{~cm}$ to $8 \mathrm{~cm}$ thickness that covers the inside radius of the 

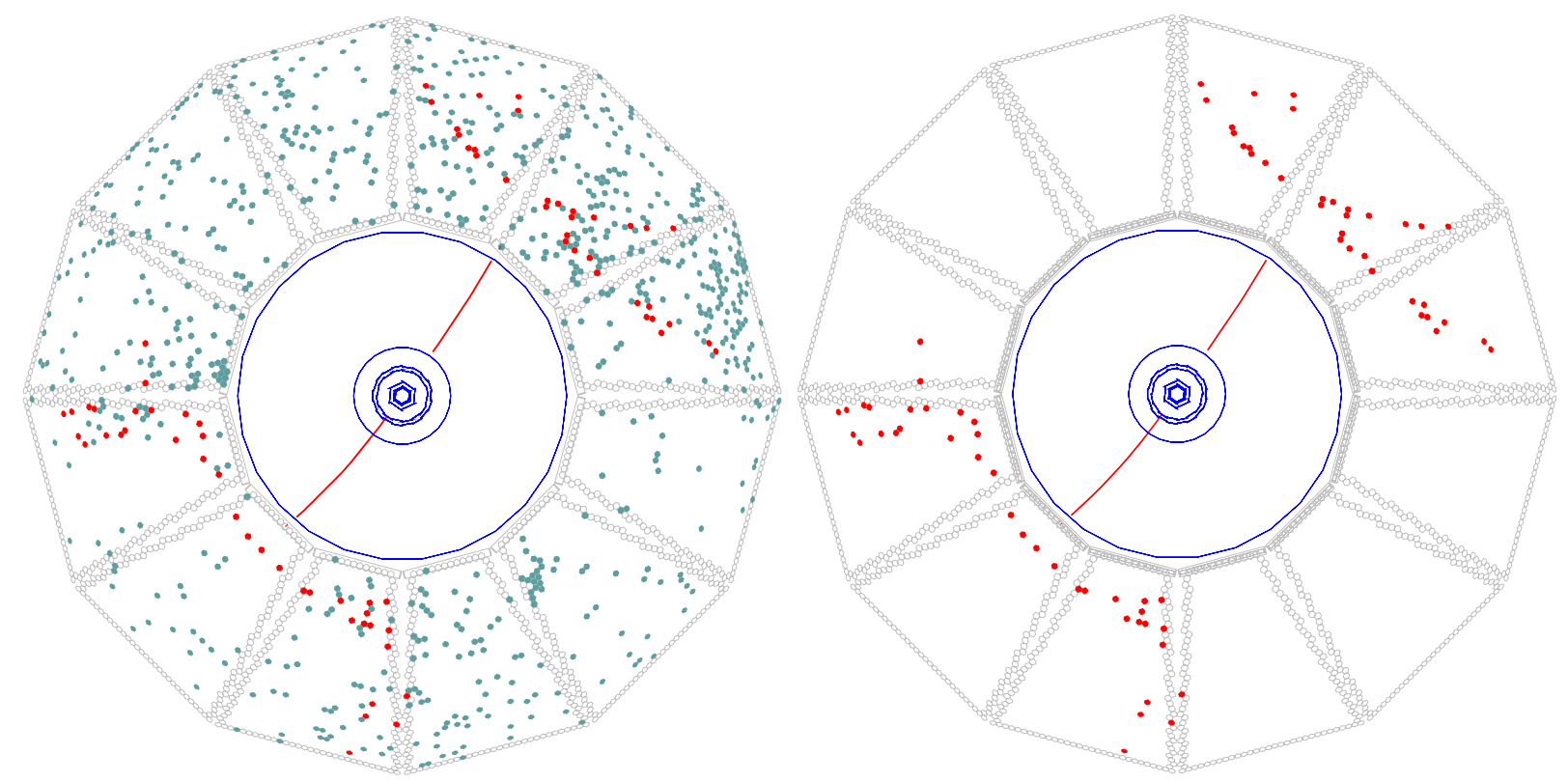

Figure 3: Display of an $e^{+} e^{-} \rightarrow \mu^{+} \mu^{-}$event reconstructed in $B A B A R$ with two different time cuts. On the left, all DiRC PMTs with signals within the \pm 300 ns trigger window are shown. On the right, only those PMTs with signals within 8 ns of the expected Cherenkov photon arrival time are displayed.

standoff box and is easily removable to facilitate access to the central detector and beam line components. Figure 4(c) shows that, at luminosities of $4.2 \times 10^{33} / \mathrm{cm}^{2} \mathrm{sec}$, the maximum scaler rates are well below the level that would cause TDC inefficiencies.

By the end of the 2001-2002 run PEP-II plans to further increase the luminosity to reach up to $5-6 \times 10^{33} / \mathrm{cm}^{2} \mathrm{sec}$. The current lead shielding should provide adequate protection from accelerator induced background until then. During the shutdown that will follow the 2001-2002 run, we plan to replace the TDCs with a faster version with deeper buffering that is designed to to have a deadtime of less than $5 \%$ at $2.5 \mathrm{MHz}$ imput rate, suitable luminosities of at least $10^{34} / \mathrm{cm}^{2} \mathrm{sec}$.

Some deterioration of the PMT front glass windows (made of B53 Borosilicate glass) that are immersed in the ultra-pure water of the standoff box has been observed, as shown in Figure 5. With water in the standoff box, these features are much less noticeable as water provides good optical coupling even to corroded glass. For most of the tubes, the observable effect is typically a slight cloudiness, but for about 50 tubes, it is much more pronounced and leads to so-called frosty PMTs. Extensive R\&D has demonstrated that the effect is associated with a loss of sodium and boron from the surface of the glass [11]. For most tubes, the leaching rate is a few microns per year, and is expected to be acceptable for the full projected ten year lifetime of the experiment. However, for the $\sim 50$ tubes, the incorrect glass was used by the manufacturer. That glass does not contain zinc, making it much more susceptible to rapid leaching. This leaching may eventually lead to either a loss of performance, or some risk of mechanical failure of the face plates for these tubes. Direct 


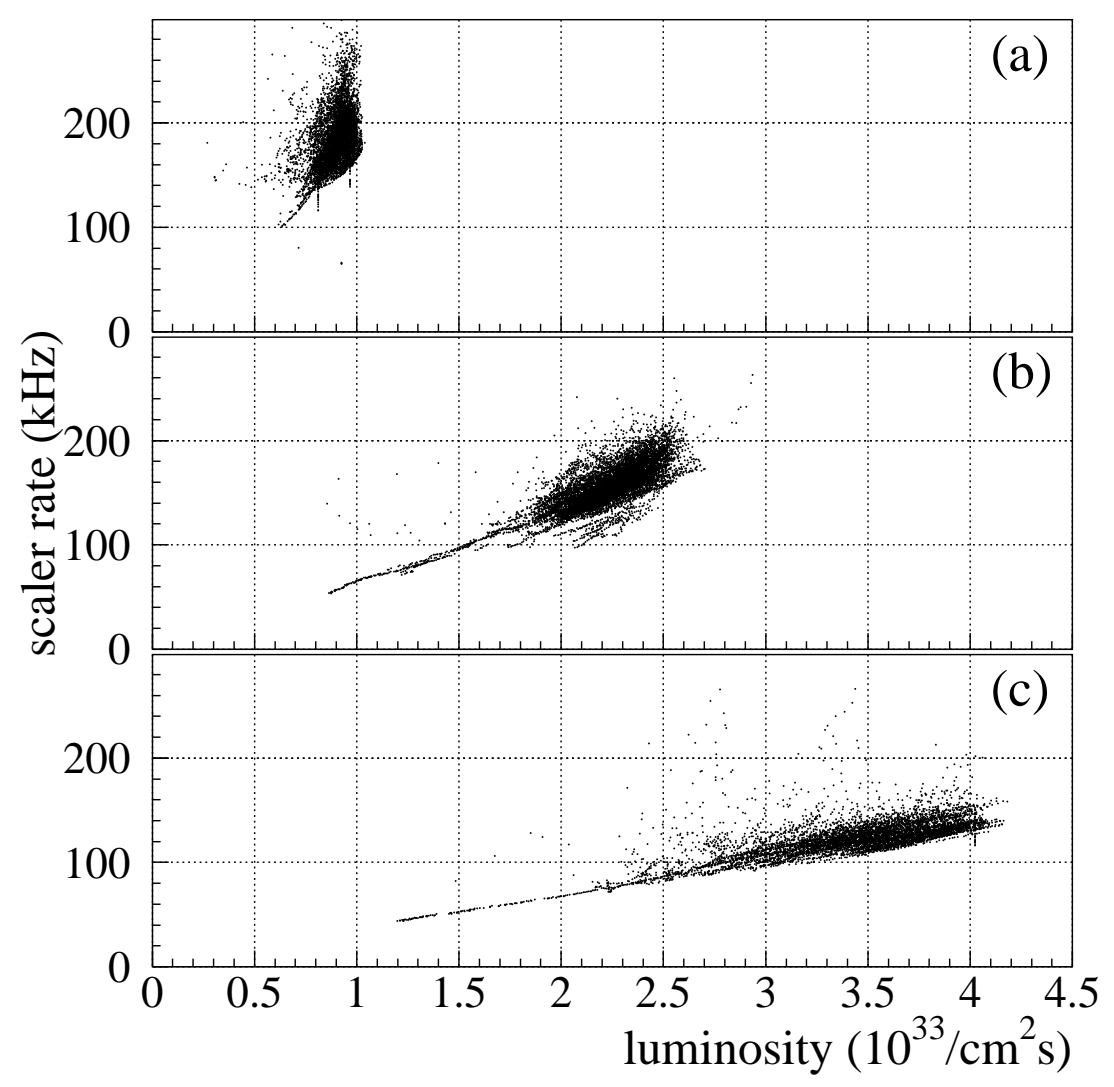

Figure 4: Maximum scaler rates measured for three different accelerator and shielding configurations in March 2000 (a), October 2000 (b) and August 2001 (c). 


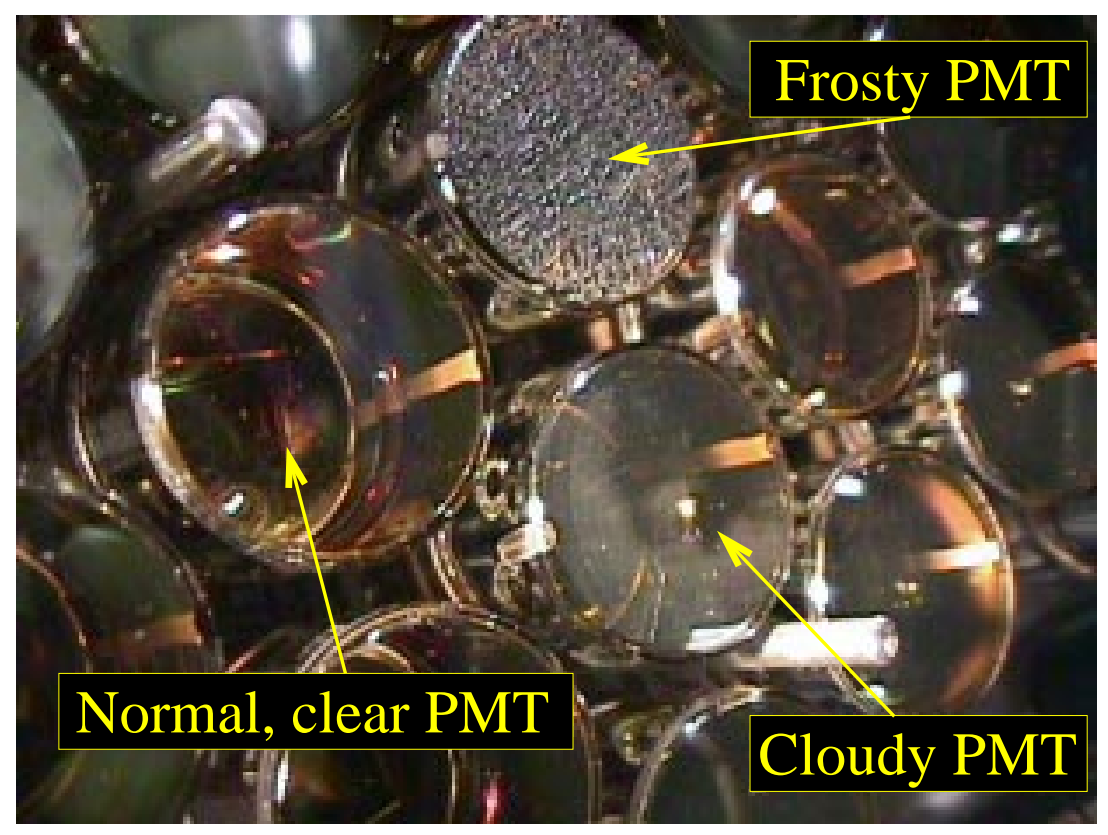

Figure 5: Example of PMT corrosion observed in October 1999, when the water was drained from the standoff box.

measurements of the number of Cherenkov photons observed in di-muon events as a function of time suggest that the total loss of photons from all sources is less than $2 \% /$ year.

After about 2.5 years of running, about $99.5 \%$ of PMTs and electronic channels are operating with nominal performance.

\section{Reconstruction}

An unbinned maximum likelihood formalism is used to incorporate all information provided by the space and time measurements from the DIRC.

The emission angle and the arrival time of the Cherenkov photons are reconstructed from the observed space-time coordinates of the PMT signals, transformed into the Cherenkov coordinate system $\left(\theta_{c}, \phi_{c}\right)$ in the following way: The known spatial position of the bar through which the track passed and the PMTs whose signal times lie within the readout window of $\pm 300 \mathrm{~ns}$ from the trigger time are used to calculate the three-dimensional vector pointing from the center of the bar end to the center of each tube. This vector is then extrapolated into the radiator bar (using Snell's law). This procedure defines, up to a 16fold ambiguity (top or bottom, left or right, forward or backward, and wedge or no-wedge reflections) the angles $\theta_{c}$ and $\phi_{c}$ of a photon.

The DIRC time measurement represents the third dimension of the photomultiplier hit reconstruction. The timing resolution is not competitive with the position information for Cherenkov angle reconstruction, but timing information is used to suppress background hits from the beam induced background and, more importantly, exclude other tracks in the same 
event as the source of the photon. Timing information is also used to resolve the forwardbackward and wedge ambiguities in the hit-to-track association.

The relevant observable used to distinguish between signal and background photons is the difference between the measured and expected photon arrival time, $\Delta t_{\gamma}$. It is calculated for each photon using the track time-of-flight (assuming it to be a charged pion), the measured time of the candidate signal in the PMT and the photon propagation time within the bar and the water filled standoff box. The time information and the requirement of using only physically possible photon propagation paths reduce the number of ambiguities from 16 to typically 3. Applying the time information also substantially improves the correct matching of photons with tracks and reduces the number of accelerator induced background signals by a factor up to 40, as illustrated in Figure 3.

The reconstruction routine currently provides a likelihood value for each of the five stable particle types $(\mathrm{e}, \mu, \pi, \mathrm{K}, \mathrm{p})$ if the track passes through the active volume of the DiRC. These likelihoods are calculated in an iterative process by maximizing the likelihood value for the entire event while testing different particle hypotheses for each track. If enough photons are found, a fit of $\theta_{c}$ is made, and the number of observed signal and background photons are calculated for each track.

\section{$5 \quad$ Results}

The parameters of expected DIRC performance were derived from extensive studies with a variety of prototypes, culminating with a full-size prototype in a beam at CERN [12]. The test beam results were well-described by Monte Carlo simulations of the detector. The performance of the full detector is close to expectations, and additional offline work, particularly on geometrical alignment, is expected to lead to further improvements.

In the absence of correlated systematic errors, the resolution $\left(\sigma_{C, t r a c k}\right)$ on the track Cherenkov angle should scale as

$$
\sigma_{C, \text { track }}=\sigma_{C, \gamma} / \sqrt{N_{p e}},
$$

where $\sigma_{C, \gamma}$ is the single photon Cherenkov angle resolution, and $N_{p e}$ is the number of photons detected. Figure 6 shows the single photon angular resolution obtained from di-muon events. There is a broad background of less than $10 \%$ relative height under the peak, that originates mostly from track-associated sources, such as $\delta$ rays, reflections off the glue-fused silica boundaries, and combinatorial background [13]. The width of the peak translates to a resolution of about $9.6 \mathrm{mr}$, in good agreement with the expected value. The measured time resolution is $1.7 \mathrm{~ns}$, close to the intrinsic $1.5 \mathrm{~ns}$ transit time spread of the PMTs.

The number of photoelectrons shown in Figure 7 varies between 16 for small values of $\left|\cos \theta_{\text {track }}\right|$ at the center of the barrel and 60 at large values of $\left|\cos \theta_{\text {track }}\right|$. This variation is well reproduced by Monte Carlo simulation, and can be understood from the geometry of the DiRc. The number of Cherenkov photons varies with the pathlength of the track in the radiator, it is smallest at perpendicular incidence at the center and increases towards the ends of the bars. In addition, the fraction of photons trapped by total internal reflection 


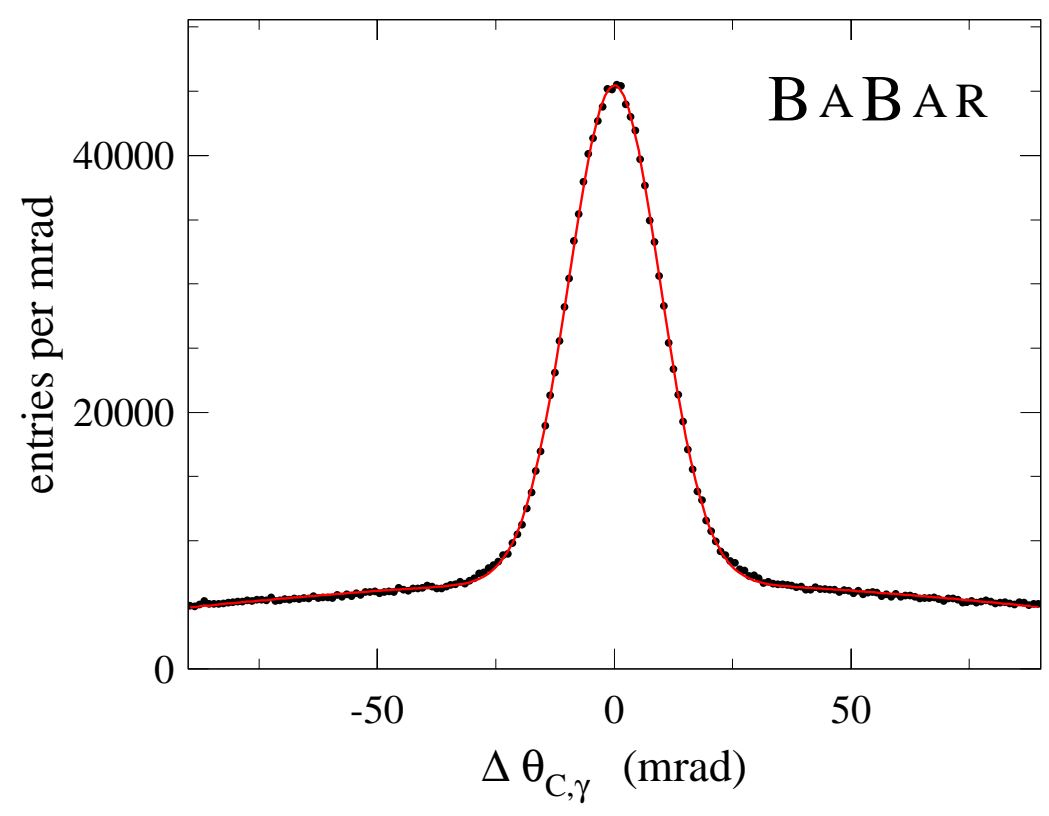

Figure 6: The difference between the measured and expected Cherenkov angle for single photons, $\Delta \theta_{c, \gamma}$ for single muons in $\mu^{+} \mu^{-}$events. The curve shows the result of a fit of two Gaussians to the data. The width of the narrow Gaussian is $9.6 \mathrm{mr}$.

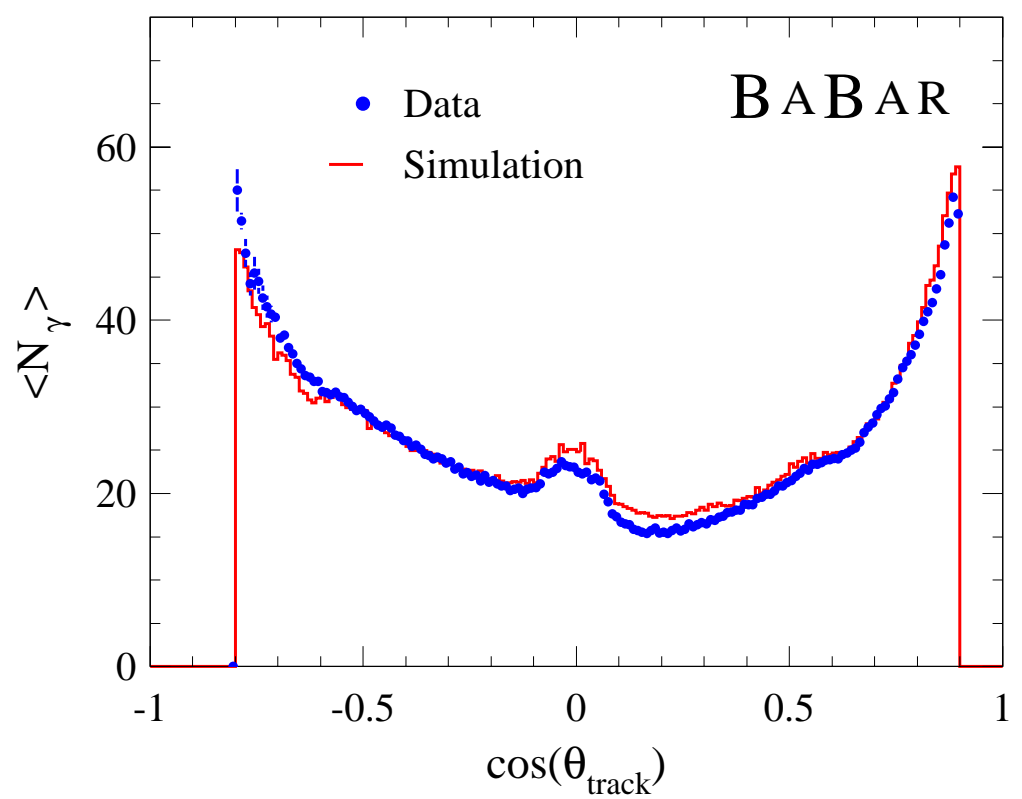

Figure 7: Number of detected photons versus track polar angle for reconstructed tracks in di-muon events compared to Monte Carlo simulation. The mean number of photons in the simulation has been tuned to match the data. 


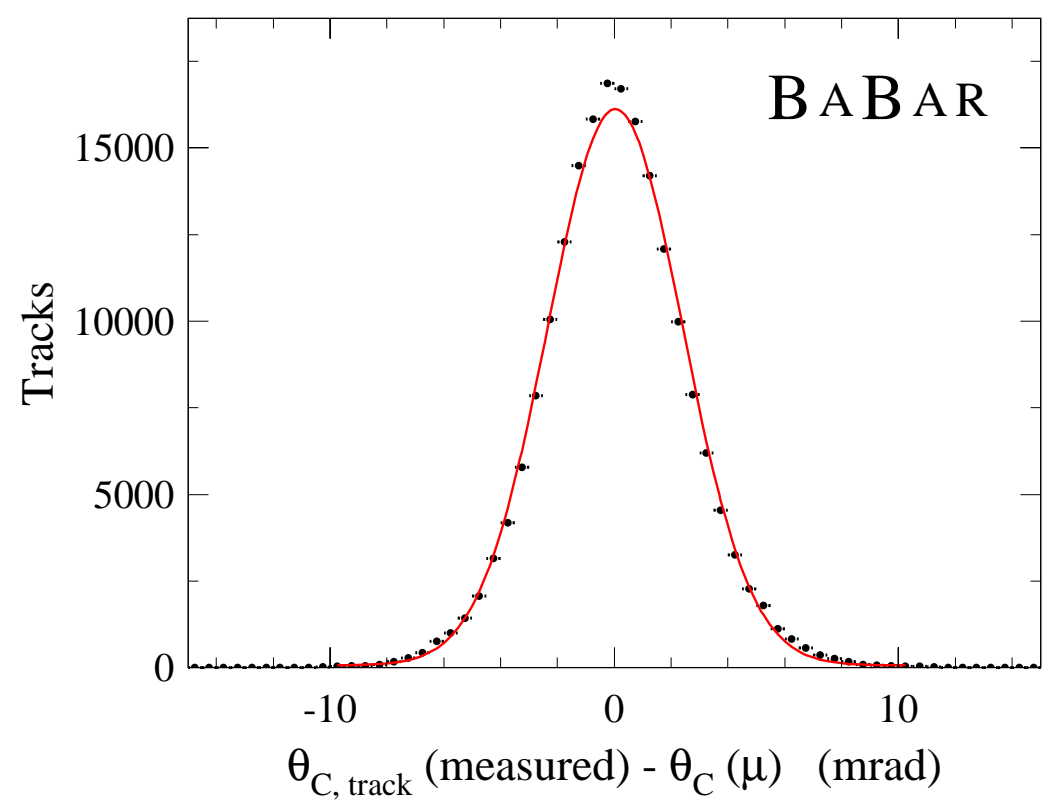

Figure 8: Resolution of the reconstructed Cherenkov polar angle per track for di-muon events. The curve shows the result of a Gaussian fit with a resolution of $2.4 \mathrm{mr}$.

rises with larger values of $\left|\cos \theta_{\text {track }}\right|$. This increase in the number of photons for forward going tracks is a good match to the increase in performance required at larger momentum.

With the present alignment, the track Cherenkov angle resolution for di-muon events is shown in Figure 8. The width of the fitted Gaussian distribution is $2.4 \mathrm{mr}$ compared to the design goal of $2.2 \mathrm{mr}$.

Figure 9 shows an example of the use of the DiRc for particle identification. The $K \pi$ invariant mass spectra are shown with and without the use of the DIRC for kaon identification. The peak corresponds to the decay of the $D^{0}$ particle.

The PID performance of the DIRC has been studied with a sample of pions and kaons, selected kinematically using $D^{0} \rightarrow K^{-} \pi^{+}$decays from inclusive $D^{*}$ production.

The pion-kaon separation power of the DIRC was defined as the difference of the expected Cherenkov angles for pions and kaons, divided by the measured track Cherenkov angle resolution. As shown in Figure 10, the separation between kaons and pions at $3 \mathrm{GeV} / c$ is about $4.4 \sigma$, within $10 \%$ of the design goal.

The efficiency for correctly identifying a charged kaon that traverses a radiator bar and the probability to wrongly identify a pion as a kaon are also determined from the inclusive $D^{*}$ sample and are shown as a function of the track momentum in Figure 11 for a particular choice of particle selection criteria. The kaon selection efficiency and pion misidentification, integrated over the $K$ and $\pi$ momentum spectra of the $D^{*}$ control sample, are $97.97 \pm 0.07 \%$ (stat. only) and $1.83 \pm 0.06 \%$ (stat. only), respectively. 


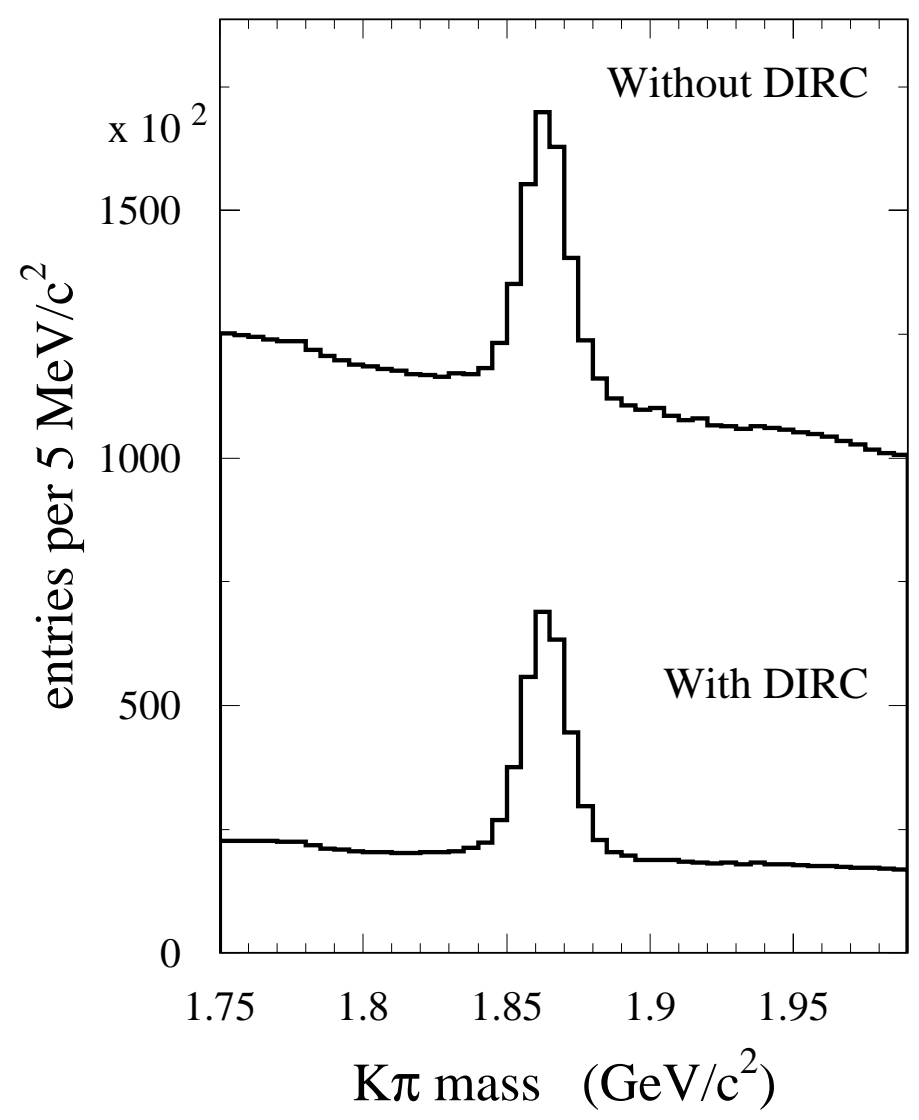

Figure 9: Invariant $\mathrm{K} \pi$ inclusive mass spectrum with and without the use of the DIRC for kaon identification. The mass peak corresponds to the decay of the $D^{0}$ particle. 


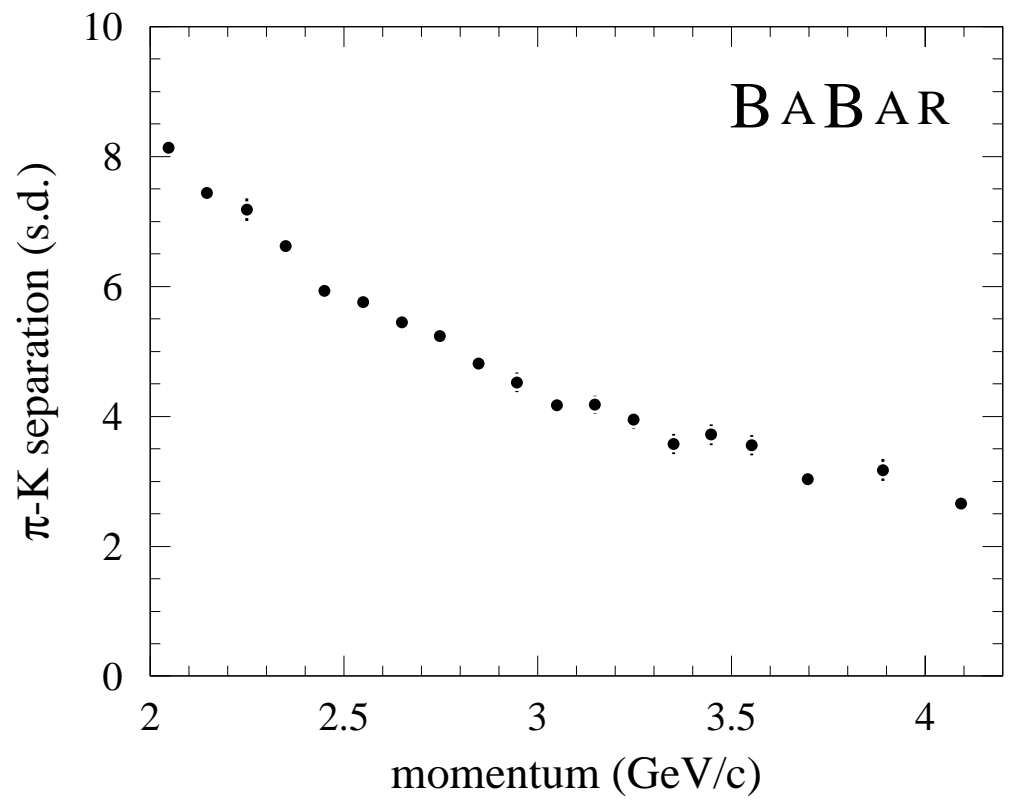

Figure 10: DiRC $\pi$-K separation versus track momentum measured in $D^{0} \rightarrow K^{-} \pi^{+}$decays selected kinematically from inclusive $D^{*}$ production.

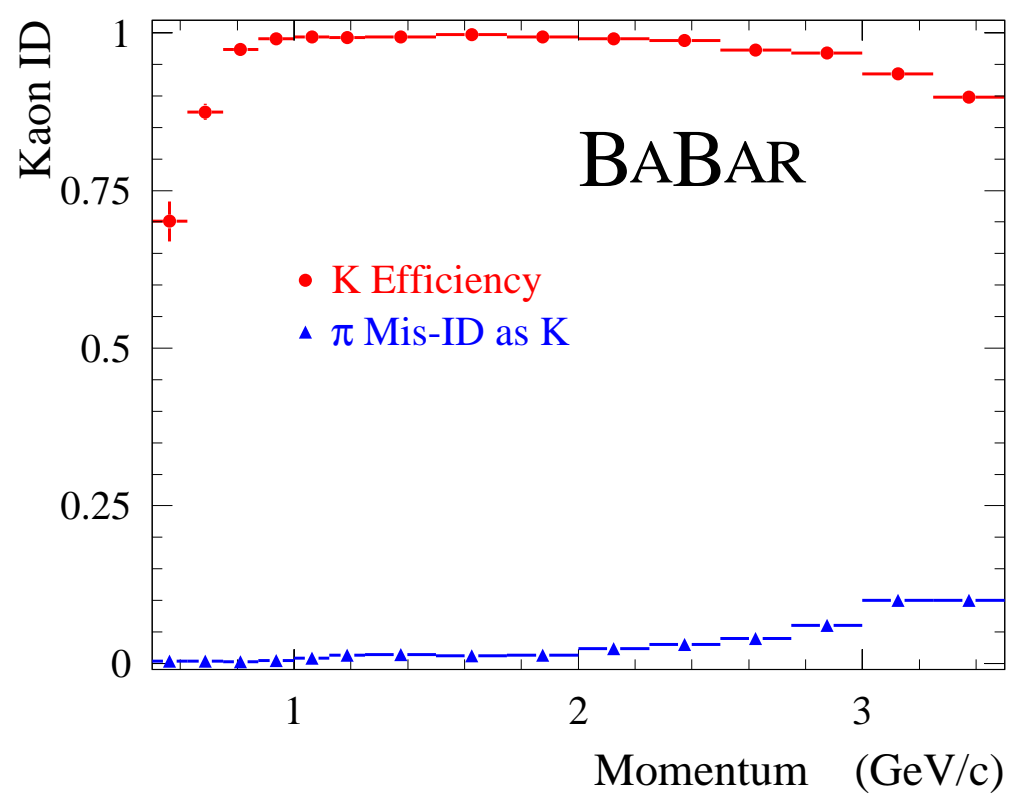

Figure 11: Efficiency and misidentification probability for the selection of charged kaons as a function of track momentum, for a particular choice of particle selection criteria. The data use $D^{0} \rightarrow K^{-} \pi^{+}$decays selected kinematically from inclusive $D^{*}$ production. 


\section{Summary}

The DIRC is a novel ring-imaging Cherenkov detector that is well-matched to the hadronic PID requirements of $B A B A R$. The DIRC has been robust and stable and, 3 years after installation, about $99.5 \%$ of all PMTs and electronic channels are still operating with nominal performance. Lead shielding in the standoff box tunnel region has been essential in reducing the sensitivity to beam-induced backgrounds at the current luminosity of $4 \times 10^{33} / \mathrm{cm}^{2} \mathrm{sec}$. At luminosities above $5-6 \times 10^{33} / \mathrm{cm}^{2} \mathrm{sec}$, the TDC chips will have to be replaced with ones of greater rate capability and deeper buffering. The design process for this is underway and the installation of the new TDCs is scheduled for the Fall 2002 shutdown of the $B_{A} B_{A R}$ experiment. The new TDCs will then prepare the DIRC for data taking at luminosities beyond $10^{34} / \mathrm{cm}^{2}$ sec.

The detector performance achieved is close to that predicted by the Monte Carlo simulations. Alignment and additional code developments are expected to further improve the performance.

\section{Acknowledgments}

Work supported by the Department of Energy under contracts DE-AC03-76SF00515 (SLAC), DE-AC03-76SF00098 (LBNL), DE-AM03-76SF0010 (UCSB), and DE-FG03-93ER40788 (CSU); the National Science Foundation grant PHY-95-11999 (Cincinnati).

\section{References}

[1] The BABAR Physics Book, Physics at an Asymmetric B Factory, P.F. Harrison, H.R. Quinn, Editors, SLAC-R-504 (1998).

[2] B.N. Ratcliff, SLAC-PUB-5946 (1992); B. N. Ratcliff, SLAC-PUB-6067 (1993); P. Coyle et al., Nucl. Instr. Methods A343 (1994) 292.

[3] Spectrosil is a trademark of TSL Group PCL, Wallsend, Tyne on Wear, NE28 6DG, England; Sold in the USA by Quartz Products Co., Louisville, KY, USA.

[4] I. Adam et al., IEEE Trans. Nucl. Sci. 45 No. 3 (June) 657; I. Adam et al., ibid 450; J. Cohen-Tanugi, M. C. Convery, B. N. Ratcliff, X. Sarazin, J. Schwiening, J. Va'vra, SLAC-JOURNAL-ICFA-21, ICFA Instrumentation Bulletin, Fall 2000 Issue.

[5] M. Benkebil et al., Nucl. Instr. Methods A442 (2000) 364.

[6] Boeing Optical Fabrication, Albuquerque, NM, USA.

[7] E. Antokhin et al., Nucl. Instr. Methods A432 (1999) 24.

[8] Electron Tubes Limited (formerly: Thorn EMI Electron Tubes), Ruislip, Middlesex, England. 
[9] P. Bourgeois, M. Karolak, G. Vasseur, Nucl. Instr. Methods A442 (2000) 105.

[10] P. Bailly et al., Nucl. Instr. Methods A433 (1999) 432.

[11] P. Bourgeois and J. Va'vra, SLAC-JOURNAL-ICFA-22, ICFA Instrumentation Bulletin, Spring 2001 Issue.

[12] R. Aleksan et al., Nucl. Instr. Methods A397 (1997) 261.

[13] A. Yarritu, S. Spanier and J. Va'vra, SLAC-PUB-9073 (2001), submitted to IEEE Trans. Nucl. Sci. 